OPEN ACCESS

Edited by: Mu-Yen Chen,

National Taichung University of Science and Technology, Taiwan

Reviewed by:

Jun-Hong Shen,

Asia University, Taiwan

Hsin-Te Wu,

National Ilan University, Taiwan

*Correspondence:

Fayong Zhao

zhaofy_72@fynu.edu.cn

Received: 27 August 2020 Accepted: 17 December 2020 Published: 15 January 2021

Citation:

Zhao F (2021) Optimal UAV's

Deployment and Transmit Power Design for Two Users Uplink NOMA

Systems.

Front. Neurorobot. 14:599344. doi: 10.3389/fnbot.2020.599344

\section{Optimal UAV's Deployment and Transmit Power Design for Two Users Uplink NOMA Systems}

\author{
Fayong Zhao* \\ School of Physics and Electronic Engineering, Fuyang Normal University, Fuyang, China
}

In order to fully utilize the spectrum resources, this work considers a unmanned aerial vehicle (UAV) uplink communication system based on non-orthogonal multiple access technology (NOMA), in which the UAV receives information from the ground users with a certain flying altitude. As an initial study, we consider a simplified setup with two ground users to draw some insightful results. Explicitly, we first formulate an optimization problem that maximizes the sum throughput subject to each user's transmit power constraint and their corresponding minimum transmission rate requirement. Then, both the optimal transmit power and UAV's deployment location are derived with the aid of employing the Karush-Kuhn-Tucher (KKT) conditions. Simulation results show that the proposed UAV's deployment scheme with the users' power allocation can achieve a higher sum throughput compared with two existing benchmark schemes.

Keywords: UAV network, uplink NOMA, power control, UAV's deployment, transmit power design

\section{INTRODUCTION}

Non-orthogonal multiple access (NOMA) is one of the key technologies for future wireless networks, which meets the heterogeneous demands on low latency, massive connectivity, high throughput, etc. (Dai et al., 2015; Ding et al., 2017). Technically, NOMA combined several modern wireless technologies, including multiple-input multiple-output (MIMO), massive MIMO and millimeter wave communications was studied in Vaezi et al. (2019) and Wang et al. (2020). Besides, the intelligent reflecting surface (IRS) aided NOMA systems were investigated in recent work (de Sena et al., 2020). The apparent benefit of NOMA which blends those compelling techniques is that it has ability in improving scalability, spectral efficiency and energy efficiency. Compared with traditional orthogonal multiple access (OMA) schemes, such as frequency division multiple access (FDMA), time division multiple access (TDMA) and code division multiple access (CDMA), NOMA simultaneously share the time, frequency and code resources. Consequently, the interuser interference is introduced actively. Notably, correct demodulation is achieved at the receiver through successive interference cancellation (SIC) (Saito et al., 2013; Chen et al., 2017). In Ding et al. (2014) and Timotheou and Krikidis (2015), downlink NOMA networks were studied, where the authors have demonstrated that NOMA can achieve better outage performance than that of OMA schemes, when both the users' rate and power allocation are carefully designed. In Zhang et al. (2016) and Al-Imari et al. (2014), uplink NOMA networks were discussed, where they showed that the uplink NOMA can improve both the spectrum efficiency and fairness index compared with OMA technique. 
The researches related to unmanned aerial vehicles (UAVs) has become a hot topic due to their wide application prospects, such as goods delivery, search and rescue, aerial photography, and telecommunications (Zeng et al., 2016). For example, Jiao et al. (2020) presented an intuitive end-to-end interaction system between a human and an UAV in which the UAV can be commanded by natural human poses. Moreover, a braininspired decision-making spiking neural network (BDM-SNN) was proposed in Zhao et al. (2018), which can help UAV making decisions in some tasks. In realistic communications, UAVs can be regarded as aerial stations for serving ground users within certain areas. In order to prolong the network lifetime, energy efficient of UAV networks was studied in Amoiralis et al. (2014), Zeng and Zhang (2017), and Zhan et al. (2018). In addition, the authors of $\mathrm{Wu}$ et al. (2019) investigated the fundamental throughput, delay, and energy tradeoffs in UAV networks. Furthermore, the authors in Wu and Zhang (2017, 2018) and $\mathrm{Wu}$ et al. (2017) pointed out that the fundamental tradeoff between the delay and the throughput in multi-user UAV networks with OMA. For reducing the access latency and improving the communication quality of UAV-based networks, it is reasonable to graft the NOMA technique into UAV networks, which is termed as UAV-enabled NOMA networks (Sharma and Kim, 2017; Cui et al., 2018; Sohail et al., 2018; Liu et al., 2019; Nasir et al., 2019; Zhao et al., 2019; Do et al., 2020). Against this background, a number of works related to the UAVenabled downlink NOMA networks have been comprehensively studied in Sharma and Kim (2017), Cui et al. (2018), and Sohail et al. (2018). Specifically, a power allocation scheme that maximizes the sum-rate of the UAV networks for reducing the energy consumption was studied in Sohail et al. (2018). In Cui et al. (2018) and Sharma and Kim (2017), the authors proposed a novel algorithm to maximize the minimum average rate by jointly optimizing the UAV's trajectory and its transmit power. Additionally, an UAV-enabled NOMA network with user pairing was studied in Nasir et al. (2019), where one user having the minimum throughput was maximized. Furthermore, to maximize the sum rate of the ground users, the authors in Liu et al. (2019) studied both the UAV's location and its transmit power. Moreover, the UAV-enabled relay NOMA networks were investigated in Do et al. (2020), and it was demonstrated that full-duplex mode can provide better outage performance than half-duplex mode. However, the extension from the downlink NOMA to uplink NOMA is not trivial because the decoding order of SIC in uplink NOMA is completely opposite to that of downlink NOMA. It is worth mentioning that the aforementioned literatures only considered the downlink scenarios, hence these existing contributions are unsuitable for the uplink scenarios, such as the data collection in the upcoming Internet of Things (IoT).

Motivated by above-mentioned reasons, this paper considers an UAV-enabled uplink NOMA with power multiplexing network, where an UAV is deployed to collect the messages transmitted from the ground users. We note that UAV-enabled uplink NOMA systems with multi-user is difficult to obtain the optimal design since the formulated optimization problem is generally difficult to tackle directly. As an initial study, similar

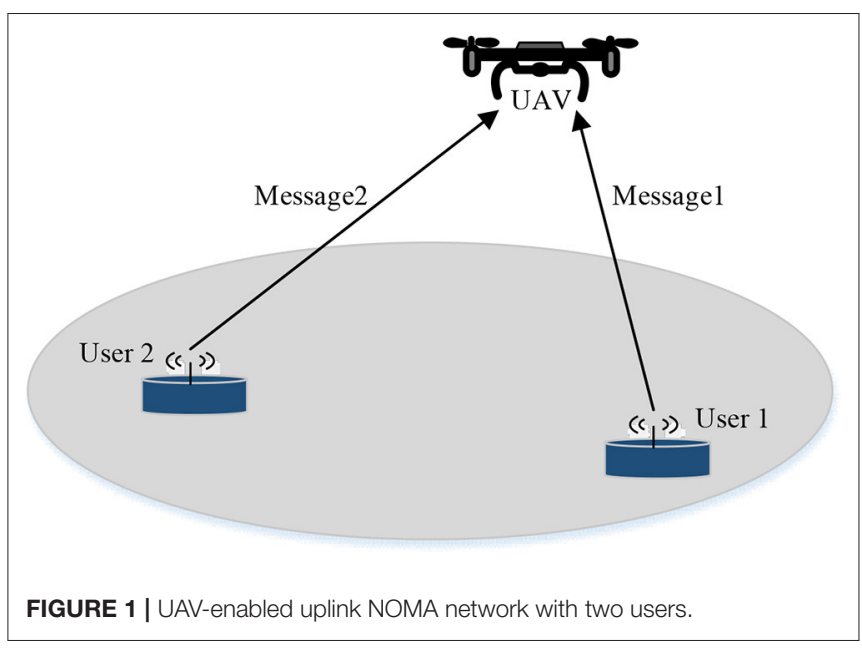

to $\mathrm{Wu}$ et al. (2018), which explores the capacity of UAVenabled/aided two user communication systems, as shown in Figure 1 we consider the optimal UAV's deployment and each user's power allocation in UAV-enabled uplink NOMA systems with two ground users to get some insightful results. Our goal is to maximize the sum rate by jointly designing the UAV's deployment location and each user's transmit power subject to the transmit power constraints and the quality of service (QoS) constraints. We should point out that our proposed algorithm is significantly different from the recent work (Duan et al., 2019) and (Du et al., 2020). Specifically, Duan et al. (2019) studies the multi-UAV aided uplink NOMA systems, where the transmit power is solved by the proposed SCA-based iterative algorithm, but the UAVs' deployment locations are not optimized. Although $\mathrm{Du}$ et al. (2020) designed the UAV deployment location, the proposed algorithm has high computational complexity and only obtains a sub-optimal solution. The main contributions of this work are summarized as follows.

- The analytical solution to the transmit power allocation policy that maximizes the sum rate for the considered dual-user systems is derived. Besides, the result can be further extended to general multi-user systems in a similar way.

- We prove that the optimal UAV deployment location lies on the line segment connected by the two users. Following this fact, the formulated optimization problem is transformed into a univariate quadratic optimization problem. Then the optimal UAV deployment location can be achieved.

- Numerical results confirm the validity of the analytical solution to the optimal UAV deployment location. In addition, our examinations demonstrate that our proposed scheme significantly outperforms the baseline schemes in terms of the sum rate.

The rest of this paper is organized as follows. In section System Model and Problem Formulation we present the system model for an UAV-enabled uplink NOMA network with two users and formulate the optimization problem. The corresponding algorithm for solving problem is introduced in section Proposed 
Algorithm for Problem (P1). In section Numerical Results, simulation results are provided to demonstrate the performance gain of our proposed algorithm. Finally, our conclusion is provided in section Conclusion.

\section{SYSTEM MODEL AND PROBLEM FORMULATION}

In this work, we consider a two user UAV-enabled uplink NOMA network, where the UAV is adopted to collect the messages transmitted from the ground users. We consider a 3-D Cartesian coordinate system where the origin is the geometric center of the two users and the $\mathrm{x}$-axis is the straight line connecting them. Assume that the distances between the two users and the origin as $D$, then the horizontal coordinates of the two users can be denoted as $\mathbf{u}_{1}=[D, 0]^{T}$ and $\mathbf{u}_{2}=[-D, 0]^{T}$, respectively. It is also assumed that the UAV flies at a fixed altitude $H$, and the horizontal coordinate of the UAV is denoted as $\boldsymbol{Q}=[x, y]^{T}$.

To capture the essential characteristics of dual-user UAVenabled NOMA systems, following Zeng et al. (2019) and the recent $3 \mathrm{GPP}$ specification ${ }^{1}$, we assume that the air-to-ground or ground-to-air channel is mainly dominated by line of sight (LoS) link. Thus, the channel gain from user $i(i=1,2)$ to the UAV is given by

$$
h_{i}=\sqrt{\frac{\eta_{0}}{\left\|Q-u_{i}\right\|^{2}+H^{2}}}, i=1,2
$$

where $\eta_{0}$ denotes the channel power gain at the reference distance $d_{0}=1 \mathrm{~m}$. Since the NOMA transmission scheme is adopted in this work, the received signal at the UAV is a series of superimposed message, which can be expressed as

$$
y=\sqrt{P_{1}} h_{1} x_{1}+\sqrt{P_{2}} h_{2} x_{2}+n
$$

where $x_{1}$ and $x_{2}$ denote the message transmitted by user 1 and user 2, respectively. $P_{1}$ and $P_{2}$ are the corresponding transmit power. $n$ denotes the zero-mean additive white Gaussian noise (AWGN) with the variance $\sigma^{2}$ at the UAV. To manage the interuser interference, the transmit power constraints are given by

$$
\begin{aligned}
& P_{1}+P_{2} \leq P_{\max } \\
& P_{i} \geq 0, i=1,2
\end{aligned}
$$

where $P_{\max }$ denotes the maximum total transmit power of the two users. For symmetry, we only consider the scenario of $x \geq 0$ in this work. Consequently, the channel gain of user 1 is greater than that of user 2. According to the principle of NOMA, the SIC is employed at the UAV to decode the messages received from different users. In particular, the UAV first decodes the message from user 1 while treating the message from user 2 as inter-user

13GPP. TR 36.777: Enhanced LTE Support for Aerial Vehicles. Available online at: https://www.3gpp.org/ftp/Specs/archive/36_series/36.777 2017. interference. Then, the decoded message from user 1 is subtracted from the superimposed received signal. Finally, the UAV decodes the message from user 2 without inter-user interference. As a result, the achievable rate of these two users can be expressed as

$$
\begin{array}{r}
R_{1}=\log _{2}\left(1+\frac{P_{1} \tilde{h}_{1}}{1+P_{2} \tilde{h}_{2}}\right) \\
R_{2}=\log _{2}\left(1+P_{2} \tilde{h}_{2}\right)
\end{array}
$$

where $\tilde{h}_{i}=\frac{h_{i}^{2}}{\sigma^{2}}=\frac{\zeta_{O}}{\left\|Q-\boldsymbol{u}_{i}\right\|^{2}+H^{2}}$, and $\zeta_{0}=\frac{\eta_{0}}{\sigma^{2}}$. As a result, the sum rate of the both users is given by

$$
R_{\text {sum }}=R_{1}+R_{2}=\log _{2}\left(1+P_{1} \tilde{h}_{1}+P_{2} \tilde{h}_{2}\right)
$$

Our goal is to maximize $R_{\max }$ by jointly optimizing the UAV deployment location and the transmit power of the both users with QoS constraints

$$
R_{i} \geq r^{*}, i=1,2
$$

where $r^{*}$ denotes the minimum rate for reliable communication. As a result, the optimization problem can be written as

$$
\begin{array}{r}
(\mathrm{P} 1): \max _{Q, P_{1}, P_{2}} R_{\text {sum }} \\
\text { s.t.(3), (7) }
\end{array}
$$

Problem $(\mathrm{P} 1)^{2}$ is a non-convex optimization problem due to the non-concavity of the objective function (8) and the nonconvexity of the constraint (7), which is, in general, difficult to solve. In the next section, we develop an algorithm to solve this problem.

\section{PROPOSED ALGORITHM FOR PROBLEM (P1)}

This section devises an algorithm to solve problem (P1) based on the solution to the transmit power of both the users. The analytical solution to problem (P1) is given as follows.

\section{Solution to Transmit Power}

Denote $Q^{*}$ as the optimal UAV deployment location and let $h_{1}^{*}$ and $h_{2}^{*}$ be the corresponding channel gains of the user 1 and user 2 , respectively. We note that the transmit power optimization problem is a convex problem, which can be efficiently solved the by Lagrangian. More explicitly, the corresponding Karush-KuhnTucher (KKT) conditions are listed as

$$
\lambda \geq 0, v_{i} \geq 0, i=1,2
$$

${ }^{2}$ In problem (P1), we assume that $x \geq 0$. However, there is also a symmetric solution to problem (P1) for $x \leq 0$, where the optimal UAV deployment is symmetric with that for $x \geq 0$ with respect to $y$-axis, and the optimal transmit power policy reverses. 


$$
\begin{gathered}
\lambda\left(P_{1}+P_{2}-P_{\max }\right)=0 \\
v_{1}\left[r^{*}-\log _{2}\left(1+\frac{P_{1} \tilde{h}_{1}^{*}}{1+P_{2} \tilde{h}_{2}^{*}}\right)\right]=0 \\
v_{2}\left[r^{*}-\log _{2}\left(1+P_{2} \tilde{h}_{2}^{*}\right)\right]=0 \\
\lambda-\frac{\left(1+v_{1}\right) \tilde{h}_{1}^{*} / \ln 2}{1+P_{1} \tilde{h}_{1}^{*}+P_{2} \tilde{h}_{2}^{*}}=0 \\
\lambda-\frac{\tilde{h}_{2}^{*} / \ln 2}{1+P_{1} \tilde{h}_{1}^{*}+P_{2} \tilde{h}_{2}^{*}}-\frac{v_{2} \tilde{h}_{2}^{*} / \ln 2}{1+P_{2} \tilde{h}_{2}^{*}} \\
+\frac{v_{1} P_{1} \tilde{h}_{1}^{*} \tilde{h}_{2}^{*} / \ln 2}{\left(1+P_{2} \tilde{h}_{2}^{*}\right)\left(1+P_{1} \tilde{h}_{1}^{*}+P_{2} \tilde{h}_{2}^{*}\right)}=0
\end{gathered}
$$

where $\lambda, v_{1}$ and $v_{2}$ are the Lagrange multipliers. As per Equation (14), we can obtain

$$
\lambda=\frac{\left(1+v_{1}\right) \tilde{h}_{1}^{*} / \ln 2}{1+P_{1} \tilde{h}_{1}^{*}+P_{2} \tilde{h}_{2}^{*}}>0
$$

According to Equation (11), we have $P_{1}+P_{2}=P_{\max }$. Upon substituting Equation (16) into Equation (15), we have

$$
\begin{aligned}
\frac{v_{2} \tilde{h}_{2}^{*}}{1+P_{2} \tilde{h}_{2}^{*}} & =\frac{\tilde{h}_{1}^{*}-\tilde{h}_{2}^{*}+\tilde{h}_{1}^{*} v_{1}}{1+P_{1} \tilde{h}_{1}^{*}+P_{2} \tilde{h}_{2}^{*}} \\
& +\frac{v_{1} P_{1} \tilde{h}_{1}^{*} \tilde{h}_{2}^{*}}{\left(1+P_{2} \tilde{h}_{2}^{*}\right)\left(1+P_{1} \tilde{h}_{1}^{*}+P_{2} \tilde{h}_{2}^{*}\right)}>0
\end{aligned}
$$

where $v_{2}>0$. As per Equation (13), we have

$$
\log _{2}\left(1+P_{2} \tilde{h}_{2}^{*}\right)=r^{*}
$$

Based on the above derivation, the optimal solution to transmit power can be expressed as

$$
\begin{array}{r}
P_{2}^{*}=\frac{2^{r^{*}}-1}{\tilde{h}_{2}^{*}} \\
P_{1}^{*}=P_{\max }-P_{2}^{*} .
\end{array}
$$

\section{Solution to UAV Deployment Location}

To determine the optimal UAV deployment location, we have the following Lemma 1.

Lemma 1. The optimal horizontal coordinate of the UAV for maximizing $R_{\text {sum }}$ should be on the line segment that linked by the two users.

Proof of Lemma 1. Assume $Q^{*}=\left[x^{*}, y^{*}\right]^{T},\left(y^{*} \neq 0\right)$, i.e. the optimal deployment location deviates from the line segment. Let us define the achievable rates of the two users at the optimal solution as $R_{1}{ }^{*}$ and $R_{2}{ }^{*}$, respectively. Then, aided with the results in the above subsection, we can obtain

$$
R_{1}^{*}=\log _{2}\left(1+\frac{P_{1}^{*} \tilde{h}_{1}^{*}}{2^{r^{*}}}\right) \geq r^{*}
$$

$$
R_{2}^{*}=\log _{2}\left(1+P_{2}^{*} \tilde{h}_{2}^{*}\right)=r^{*}
$$

However, if we deploy the UAV at $Q^{\prime}=\left[x^{*}, 0\right]^{T}$, the corresponding channel gains $\tilde{h}_{1}{ }^{\prime}$ and $\tilde{h}_{2}{ }^{\prime}$ will be larger than $\tilde{h}_{1}^{*}$ and $\tilde{h}_{2}^{*}$, respectively. Assuming that $P_{1}^{\prime}$ and $P_{2}^{\prime}$ are the optimal transmit power at this time, then we have

$$
\begin{aligned}
& R_{1}{ }^{\prime}=\log _{2}\left(1+\frac{P_{1}{ }^{\prime} \tilde{h}_{1}{ }^{\prime}}{2^{r^{*}}}\right)^{(a)} \log _{2}\left(1+\frac{P_{1}^{*} \tilde{h}_{1}^{*}}{2^{r^{*}}}\right)=R_{1}^{*} \geq r^{*} \\
& R_{2}{ }^{\prime}=\log _{2}\left(1+P_{2}{ }^{\prime} \tilde{h}_{2}{ }^{\prime}\right)=r^{*}=R_{2}^{*}
\end{aligned}
$$

where (a) holds since $P_{2}{ }^{\prime}=\frac{2^{r^{*}}-1}{\tilde{h}_{2}{ }^{\prime}}<P_{2}{ }^{*}$, and thus having $P_{1}{ }^{\prime}=$ $P_{\max }-P_{2}^{\prime}>P_{\max }-P_{2}^{*}=P_{1}^{*}$. As a result, $Q^{\prime}=\left[x^{*}, 0\right]^{T}$ is also a feasible deployment location. Apparently, the corresponding sum rate will be larger than that at $Q^{*}$.

Similarly, if $x^{*}>D$, we can deploy the UAV at $Q^{\prime \prime}=[D, 0]^{T}$, and the corresponding sum rate is larger. Consequently, the optimal UAV deployment location has to be located at one point on the line segment linked by the two users. This completes the proof.

Based on the above results, problem (P1) can be further reformulated as

$$
\begin{array}{r}
(\mathrm{P} 2): \max _{x^{*}} \phi\left(x^{*}\right) \\
\text { s.t. } \leq x^{*} \leq D
\end{array}
$$

where

$$
\begin{aligned}
& \phi\left(x^{*}\right)=P_{1}^{*} \tilde{h}_{1}^{*}=\frac{\zeta_{0} P_{\max }}{H^{2}+\left(D-x^{*}\right)^{2}} \\
& -\frac{\left(2^{r^{*}}-1\right)\left[H^{2}+\left(D-x^{*}\right)^{2}\right]}{H^{2}+\left(D+x^{*}\right)^{2}}
\end{aligned}
$$

Clearly, problem (P2) is a univariate quadratic optimization problem. The derivative of $\phi\left(x^{*}\right)$ is given by

$$
\frac{d \phi\left(x^{*}\right)}{d x^{*}}=\frac{2\left[2 D-\left(x^{*}\right)^{2}-\Gamma x^{*}+\Gamma D-2 D\left(H^{2}+D^{2}\right)\right]}{\left[H^{2}+\left(D-x^{*}\right)^{2}\right]^{2}}
$$

where $\Gamma=\frac{\zeta_{0} P_{\max }}{2^{r^{*}}-1}$. We note that $\frac{d \phi\left(x^{*}\right)}{d x^{*}}$ is equivalent to

$$
2 D\left(x^{*}\right)^{2}-\Gamma x^{*}+\Gamma D-2 D\left(H^{2}+D^{2}\right)=0
$$

It can be observed that Equation (29) is a quadric equation. To proceed, we define $\Delta=\Gamma^{2}-8 \Gamma D^{2}+16 D^{2}\left(H^{2}+D^{2}\right)$. If $\Delta<0$, $\phi\left(x^{*}\right)$ is a monotonically increasing function with respect to $x^{*}$, and therefore the optimal deployment location is arrived when $x_{o p t}=D$. By contrast, as $\Delta \geq 0$, the two stationary points $x_{1}^{*}$ and $x_{2}^{*}$ can be derived as

$$
x_{1}^{*}=\frac{\Gamma-\sqrt{\Delta}}{4 D}
$$




$$
x_{2}^{*}=\frac{\Gamma+\sqrt{\Delta}}{4 D}
$$

Based on the relation between $x_{1}^{*}, x_{2}^{*}$ and the interval $(0, D)$, the solution to the optimal UAV deployment location $x_{\text {opt }}$ can be obtained via Algorithm 1. Finally, we have to check these obtained solutions until finding the one meets the condition of $R_{1}^{*} \geq r^{*}$.

Algorithm 1 Solution to the optimal UAV deployment location. Input: $D, H, r^{*}, P_{\max }$, and $\zeta_{0}$.

1: Calculate $x_{1}^{*}$ and $x_{2}^{*}$ according to Equations (30) and (31), respectively.

2: If $x_{1}^{*} \in(0, D), x_{2}^{*} \in(0, D)$, then $x_{\mathrm{opt}}=$ $\arg \max \left\{\phi(0), \phi\left(x_{1}^{*}\right), \phi\left(x_{2}^{*}\right), \phi(D)\right\}$.

3: If $x_{1}^{*} \in(0, D), x_{2}^{*} \notin(0, D)$, then $x_{\mathrm{opt}}=$ $\arg \max \left\{\phi(0), \phi\left(x_{1}^{*}\right), \phi(D)\right\}$.

4: If $x_{1}^{*} \notin(0, D), x_{2}^{*} \in(0, D)$, then $x_{\text {opt }}=\arg \max \{\phi(0), \phi(D)\}$.

5: If $x_{1}^{*} \notin(0, D), x_{2}^{*} \notin(0, D)$, then $x_{\mathrm{opt}}=\arg \max \{\phi(0), \phi(D)\}$.

Output: The optimal UAV deployment location $x_{\text {opt }}$.

\section{Solution to Problem (P1)}

According to the results of the previous two subsections, the optimal solution to problem (P1) can be obtained via Algorithm 2 given below, and the flow chart of the proposed algorithm is shown in Figure 2. Specifically, the optimal UAV deployment location is first obtained via Algorithm 1. Then, the corresponding channel gains of user 2 is calculated. Finally, the optimal solution to the transmit power can be obtained based on Equations (19) and (20). It is easy to calculate that, in the worst case, the proposed algorithm requires 59 multiplications and 44 additions.

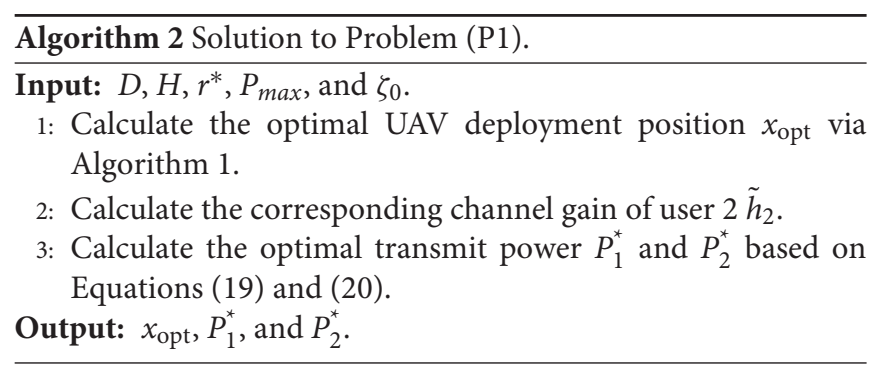

\section{NUMERICAL RESULTS}

In this section, simulation results are provided to demonstrate the effectiveness of our proposed algorithm (denoted as N-LPJO). Referring to the existing the literatures (Wu and Zhang, 2017, 2018; Wu et al., 2017, 2019; Zeng and Zhang, 2017; Cui et al., 2018; Sohail et al., 2018; Zhan et al., 2018), the simulation parameters, unless otherwise specified, are set as: the maximum total transmit power of the two users $P_{\max }=10 \mathrm{dBm}$, the UAV altitude $H=200 \mathrm{~m}$, the distance between the two users and the

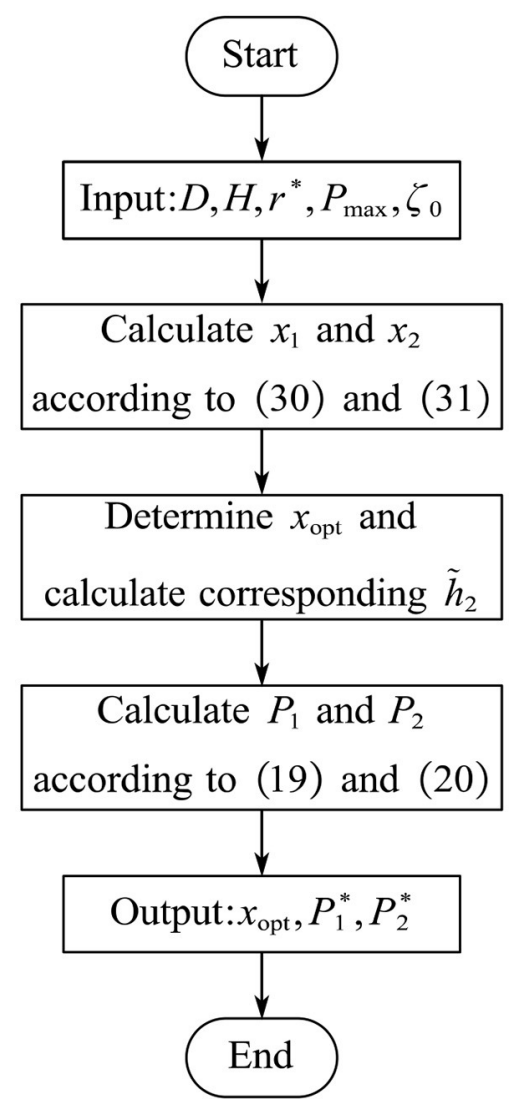

FIGURE 2 | Flow chart of the proposed algorithm.

origin $D=400 \mathrm{~m}$, and the reference signal-to-noise ratio (SNR) $\zeta_{0}=80 \mathrm{~dB}$.

For comparison, the following three baseline schemes are invoked:

1) FDMA: The UAV collects the messages in FDMA manner, where both the UAV deployment location and transmit power are jointly optimized;

2) N-FLPO: The scheme in Duan et al. (2019), where only the transmit power is optimized while the UAV is fixed at the geometric center of two users, i.e., $[0,0]^{T}$;

3) N-LOFP: The UAV collects the messages in NOMA manner, where only the UAV deployment location is optimized while the transmit power is fixed as $P_{1}=2 \mathrm{~mW}$ and $P_{2}=8 \mathrm{~mW}$.

Figure 3 plots the optimal UAV deployment location of the NLPJO scheme vs. $r^{*}$, where the numerical results (obtained by 1-D search method) are invoked to reveal the optimality of our proposed algorithm. It can be noted that the sum rates achieved by $1-\mathrm{D}$ search method meets that arrived by our developed analytical solution. This phenomenon implies the optimality of the analytical solution. We also observe that the optimal UAV deployment location is close to user 1 , which is beneficial for improving the achievable rate of user 1 . Moreover, we observe that the optimal UAV deployment location moves toward the 


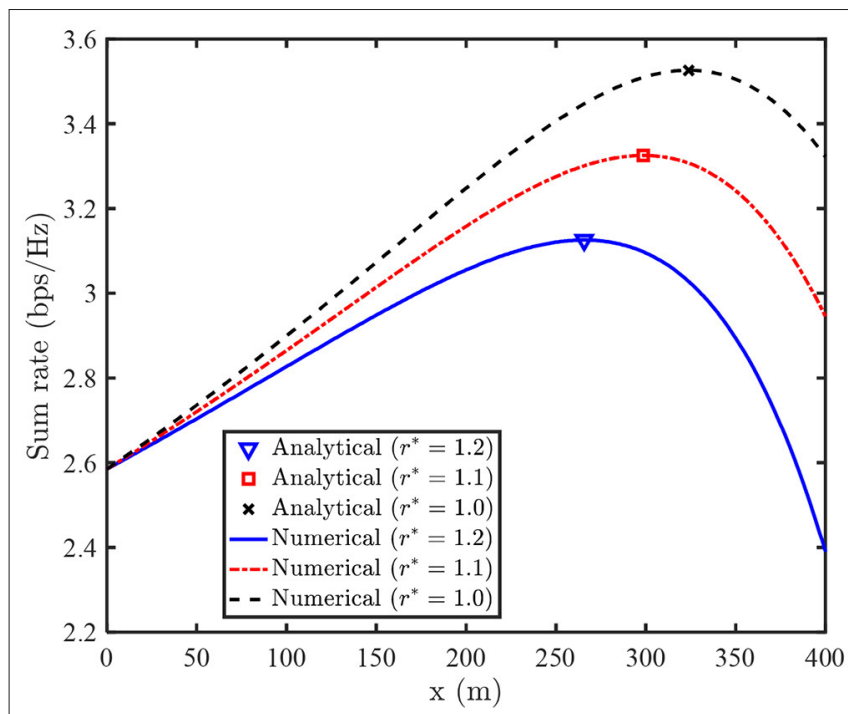

FIGURE 3 | Optimal UAV's deployment location for N-LPJO scheme vs. $r^{*}$.

origin as $r^{*}$ increases. This is due to the fact that it will cost less transmit power to meet user 2's QoS constraint if the UAV is deployed close to the origin.

Figure 4 plots the achievable sum rates of the four different schemes vs. $r^{*}$. We observe that the sum rates of the N-LPJO and the FDMA schemes decreases as $r^{*}$ increases. This is mainly because the poorer user has to increase the transmit power for meeting QoS requirement. Meanwhile, the stronger user has to decrease its transmit power. However, the sum rate of the $\mathrm{N}$ FLPO scheme remains unchanged regardless of $r^{*}$, this is due to the fact that $\tilde{h}_{1}=\tilde{h}_{2}$ and thus $R_{\text {sum }}=\log _{2}\left(1+P_{1} \tilde{h}_{1}+\right.$ $\left.P_{2} \tilde{h}_{2}\right)=\log _{2}\left(1+\left(P_{1}+P_{2}\right) \tilde{h}_{2}\right)=\log _{2}\left(1+P_{\max } \tilde{h}_{2}\right)$ is a constant. Besides, different from the other three schemes, the sum rate of the N-LOFP scheme remains unchanged when $r^{*} \leq 1.1$ bps and decreases when $r^{*}=1.2$ bps. This is because the optimal UAV deployment location can naturally meet the QoS constraints if the QoS constraints are not very tight. It is clearly shown that our proposed N-LPJO scheme outperforms both the NFLPO scheme and the N-LOFP scheme, which demonstrates the necessity of optimizing the UAV deployment position and the transmit power, respectively. Furthermore, our proposed NLPJO scheme also outperforms the FDMA scheme since NOMA can provide higher spectral efficiency than OMA.

Figure 5 shows that the sum rate of our proposed N-LPJO scheme vs. the distance $D$ between the two users and the origin. Firstly, we observe that the sum rate decreases as $D$ increases for each QoS requirement $r^{*}$. This can be explained as: the channel gain of user 2 sharply decreases once $D$ increases. As a result, user 2 has to improve the transmit power to meet the minimum rate requirement, while user 1 has to decrease its transmit power, thus leading to the decrease in the sum rate. Secondly, we note that the difference of the sum rate achieved by the different $r^{*}$ becomes apparent with the increasing of $D$.

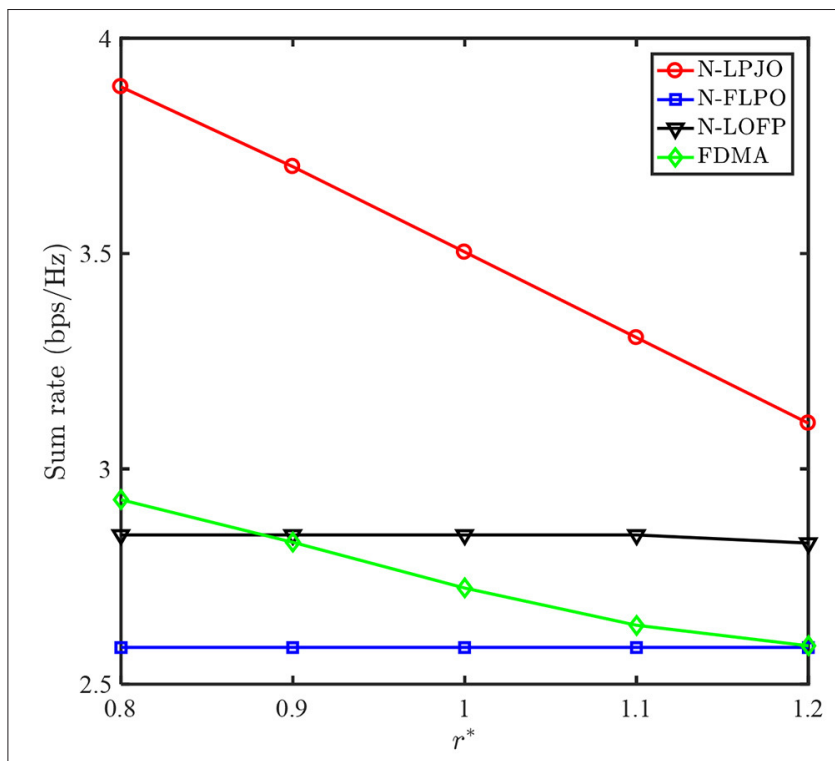

FIGURE 4 | Sum rates of four schemes vs. $r^{*}$.

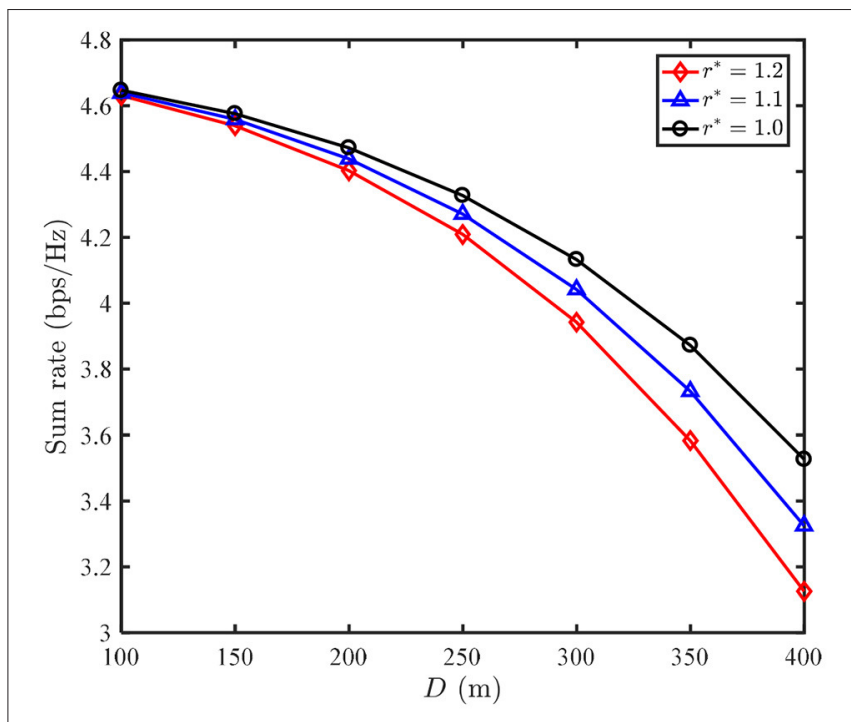

FIGURE 5 | Sum rate of N-LPJO scheme vs. distance D between both users.

Figure 6 shows the sum rate of our proposed N-LPJO scheme vs. UAV's altitude $H$. It is observed that all the sum rates decrease as $H$ increases for each QoS requirement. This is due to the fact that both the channel gains of the two users decrease as $H$ increases, hence user 2 has to increase its transmit power for satisfying the QoS requirement, leading to the received power from user 1 decreases. Additionally, we observe that all the performance gains attained by the different $r^{*}$ decrease as $H$ increases. This is because that the effect of $H$ on the two users' channel gains will be rather small as $H$ becomes sufficiently large. 


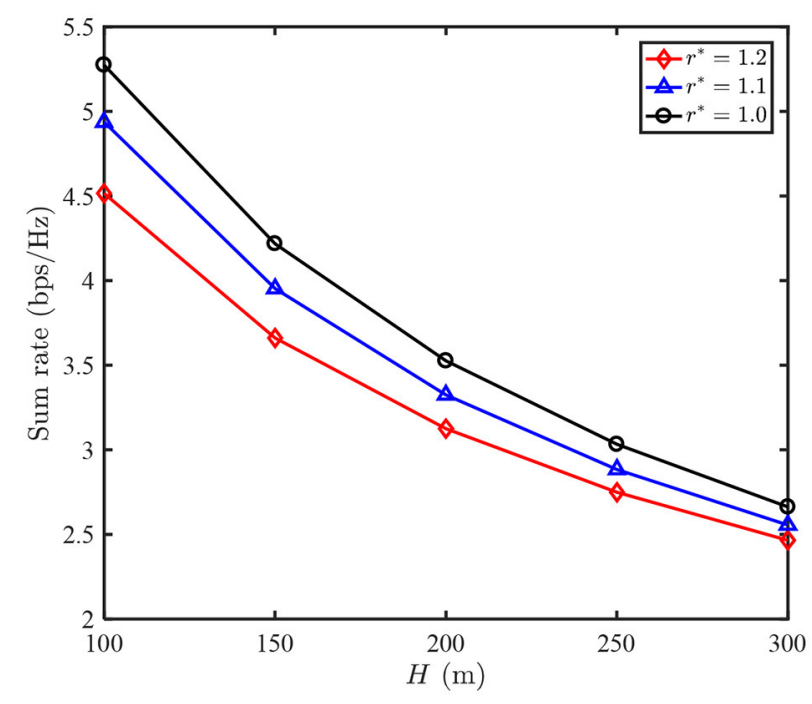

FIGURE 6 | Sum rate of N-LPJO scheme vs. UAV altitude $H$.

\section{CONCLUSIONS}

In this paper, we have investigated an UAV-enabled uplink NOMA system under a total power constraint. To maximize the sum rate of two users, we have demonstrated that the UAV should be deployed at a certain point over the line segment linked by the two users. Then, we have translated the corresponding optimization into a univariate quadratic optimization problem,

\section{REFERENCES}

Al-Imari, M., Xiao, P., Imran, M. A., and Tafazolli, R. (2014). "Uplink non-orthogonal multiple access for 5G wireless networks," in 2014 11th International Symposium on Wireless Communications Systems (Barcelona), 781-785. doi: 10.1109/ISWCS.2014.6933459

Amoiralis, E. I., Tsili, M. A., Spathopoulos, V., and Hatziefremidis, A. (2014). Energy efficiency optimization in UAVs: a review. Mater. Sci. Forum 792, 281-286. doi: 10.4028/www.scientific.net/MSF.792.281

Chen, Z., Ding, Z., Dai, X., and Zhang, R. (2017). An optimization perspective of the superiority of NOMA compared to conventional OMA. IEEE Trans. Signal Process. 65, 5191-5202. doi: 10.1109/TSP.2017.2725223

Cui, F., Cai, Y., Qin, Z., Zhao, M., and Li, G. Y. (2018). "Joint trajectory design and power allocation for UAV-enabled non-orthogonal multiple access systems," in 2018 IEEE Global Communications Conference (GLOBECOM) (Abu Dhabi), 1-6. doi: 10.1109/GLOCOM.2018.8647352

Dai, L., Wang, B., Yuan, Y., Han, S. I. C., and Wang, Z. (2015). Non-orthogonal multiple access for 5G: solutions, challenges, opportunities, and future research trends. IEEE Commun. Mag. 53, 74-81. doi: 10.1109/MCOM.2015.7263349

de Sena, A. S., Carrillo, D., Fang, F., Nardelli, P. H. J., Costa, D. B., Dias, U. S., et al. (2020). What role do intelligent reflecting surfaces play in multiantenna non-orthogonal multiple access? IEEE Wireless Commun. 27, 24-31. doi: 10.1109/MWC.001.2000061

Ding, Z., Lei, X., Karagiannidis, G. K., Schober, R., and Bhargava, V. A. (2017). Survey on non-orthogonal multiple access for $5 \mathrm{G}$ networks: research challenges and future trends. IEEE J. Sel. Areas Commun. 35, 2181-2195. doi: 10.1109/JSAC.2017.2725519 which can be efficiently solved by our developed scheme. Simulation results showed that our proposed scheme significantly outperforms both the two benchmarks in terms of the sum rate. It should be pointed out that the proposed power allocation policy can be extended to general multi-user systems in a similar way, but extending the proposed UAV deployment scheme to general multi-user systems is not trivial. Our future works will commit to solving the sum rate maximization problem in general multi-user networks.

\section{DATA AVAILABILITY STATEMENT}

The raw data supporting the conclusions of this article will be made available by the authors, without undue reservation.

\section{AUTHOR CONTRIBUTIONS}

The author confirms being the sole contributor of this work and has approved it for publication.

\section{FUNDING}

This research was funded in part by the Natural Science Research Project of Education Department of Anhui Province of China under Grant KJ2019A1002, in part by horizontal cooperation project under Grant XDHX201705, and in part by the Key scientific and technological projects of Anhui Province under Grand 1501031114.

Ding, Z., Yang, Z., Fan, P., and Poor, H. V. (2014). On the performance of nonorthogonal multiple access in $5 \mathrm{G}$ systems with randomly deployed users. IEEE Signal Process. Lett. 21, 1501-1505. doi: 10.1109/LSP.2014.2343971

Do, D., Nguyen, T., Le, C., Voznak, M., Kaleem, Z., and Rabie, K. M. (2020) UAV relaying enabled NOMA network with hybrid duplexing and multiple antennas. IEEE Access 8, 186993-187007. doi: 10.1109/ACCESS.2020.30 30221

Du, J., Wang, Z., Fan, Z., and Wan, X. (2020). "Sum rate maximization for UAVenabled wireless powered NOMA systems," in 2020 IEEE/CIC International Conference on Communications in China (ICCC) (Chongqing), 753-757. doi: 10.1109/ICCC49849.2020.9238850

Duan, R., Wang, J., Jiang, C., Yao, H., Ren, Y., and Qian, Y. (2019). Resource allocation for multi-UAV aided IoT NOMA uplink transmission systems. IEEE Internet Things J. 6, 7025-7037. doi: 10.1109/JIOT.2019.2913473

Jiao, R., Wang, Z., Chu, R., Dong, M., Rong, Y., and Chou, W. (2020). An intuitive end-to-end human-UAV interaction system for field exploration. Front. Neurorobot. 13:117. doi: 10.3389/fnbot.2019.00117

Liu, X., Wang, J., Zhao, N., Chen, Y., Zhang, S., Ding, Z., et al. (2019). Placement and power allocation for NOMA-UAV networks. IEEE Wireless Commun. Lett. 8, 965-968. doi: 10.1109/LWC.2019.2904034

Nasir, A. A., Tuan, H. D., Duong, T. Q., and Poor, H. V. (2019). UAVenabled communication using NOMA. IEEE Trans. Commun. 67, 5126-5138. doi: 10.1109/TCOMM.2019.2906622

Saito, Y., Kishiyama, Y., Benjebbour, A., Nakamura, T., Li, A., and Higuchi, K. (2013). "Non-orthogonal multiple access (NOMA) for cellular future radio access," in 2013 IEEE 77th Vehicular Technology Conference (VTC Spring) (Dresden), 1-5. doi: 10.1109/VTCSpring.2013.6692652 
Sharma, P. K., and Kim, D. I. (2017). "UAV-enabled downlink wireless system with non-orthogonal multiple access," in IEEE Globecom Workshops (GC Wkshps) (Singapore), 1-6. doi: 10.1109/GLOCOMW.2017.8269066

Sohail, M. F., Leow, C. Y., and Won, S. (2018). Non-orthogonal multiple access for unmanned aerial vehicle assisted communication. IEEE Access 6, 22716-22727. doi: 10.1109/ACCESS.2018.2826650

Timotheou, S., and Krikidis, I. (2015). Fairness for non-orthogonal multiple access in 5G systems. IEEE Signal Process. Lett. 22, 1647-1651. doi: 10.1109/LSP.2015.2417119

Vaezi, M., Amarasuriya, G., Liu, Y., Arafa, A., Fang, F., and Ding, Z. (2019). Interplay between NOMA and other emerging technologies: a survey. IEEE Trans. Cogn. Commun. Netw. 5, 900-919. doi: 10.1109/TCCN.2019.2933835

Wang, Y., Tian, Z., and Cheng, X. (2020). Enabling technologies for spectrum and energy efficient NOMA-MmWave-MaMIMO systems. IEEE Wireless Commun. 7, 53-59. doi: 10.1109/MWC.001.2000055

Wu, Q., Liu, L., and Zhang, R. (2019). Fundamental trade-offs in communication and trajectory design for UAV-enabled wireless network. IEEE Wireless Commun. 26, 36-44. doi: 10.1109/MWC.2018.1800221

Wu, Q., Xu, J., and Zhang, R. (2018). Capacity characterization of UAV-enabled two-user broadcast channel. IEEE J. Sel. Areas Commun. 36, 1955-1971. doi: 10.1109/JSAC.2018.2864421

Wu, Q., Zeng, Y., and Zhang, R. (2017). "Joint trajectory and communication design for UAV-enabled multiple access," in LOBECOM 2017 2017 IEEE Global Communications Conference (Singapore), 1-6. doi: 10.1109/GLOCOM.2017.8254949

Wu, Q., and Zhang, R. (2017). "Delay-constrained throughput maximization in UAV-enabled OFDM systems," in 2017 23rd Asia-Pacific Conference on Communications (APCC) (Perth, WA), 1-6. doi: 10.23919/APCC.2017.83 04088

$\mathrm{Wu}$, Q., and Zhang, R. (2018). Common throughput maximization in UAVenabled OFDMA systems with delay consideration. IEEE Trans. Commun. 66, 6614-6627. doi: 10.1109/TCOMM.2018.2865922
Zeng, Y., Wu, Q., and Zhang, R. (2019). Accessing from the sky: a tutorial on UAV communications for 5G and beyond. Proc. IEEE 107, 2327-2375. doi: 10.1109/JPROC.2019.2952892

Zeng, Y., and Zhang, R. (2017). Energy-efficient UAV communication with trajectory optimization. IEEE Trans. Wireless Commun. 16, 3747-3760. doi: 10.1109/TWC.2017.2688328

Zeng, Y., Zhang, R., and Lim, T. J. (2016). Wireless communications with unmanned aerial vehicles: opportunities and challenges. IEEE Commun. Mag. 54, 36-42. doi: 10.1109/MCOM.2016.7470933

Zhan, C., Zeng, Y., and Zhang, R. (2018). Energy-efficient data collection in UAV enabled wireless sensor network. IEEE Wireless Commun. Lett. 7, 328-331. doi: 10.1109/LWC.2017.2776922

Zhang, N., Wang, J., Kang, G., and Liu, Y. (2016). Uplink non-orthogonal multiple access in 5G systems. IEEE Commun. Lett. 20, 458-461. doi: 10.1109/LCOMM.2016.2521374

Zhao, F., Zeng, Y., and Xi, B. (2018). A brain-inspired decision-making spiking neural network and its application in unmanned aerial vehicle. Front. Neurorobot. 12:56. doi: 10.3389/fnbot.2018.00056

Zhao, N., Pang, X., Li, Z., Chen, Y., Li, F., Ding, Z., et al. (2019). Joint trajectory and precoding optimization for UAV-assisted NOMA networks. IEEE Trans. Commun. 67, 3723-3735. doi: 10.1109/TCOMM.2019.2895831

Conflict of Interest: The author declares that the research was conducted in the absence of any commercial or financial relationships that could be construed as a potential conflict of interest.

Copyright (C) 2021 Zhao. This is an open-access article distributed under the terms of the Creative Commons Attribution License (CC BY). The use, distribution or reproduction in other forums is permitted, provided the original author(s) and the copyright owner(s) are credited and that the original publication in this journal is cited, in accordance with accepted academic practice. No use, distribution or reproduction is permitted which does not comply with these terms. 